\title{
La Escritura Como Traducción: Tres Tristes Tigres y una Cobra
}

"Porque siendo assi que la mayor parte de la gracia y gentileza de la lengua castellana consiste en hablar por metáforas, atándose el que traduze a no poner más de lo que halla escrito en la lengua de que traduze, tiene grandissima dificultad en dar al castellano la gracia y lustre que, escriviendo de su cabeça, le daría."

Juan de Valdés, Diálogo de la Lengua (1539)

I.

Al discutir las contradicciones en que incurre Freud al intentar traducir los sueños al tiempo que admite la imposibilidad de traducirlos, Jacques Derrida observa:

Translation, a system of translation, is possible only if a permanent code allows a substitution or transformation of signifiers while retaining the same signified, always present, despite the absence of any specific signifier.

("Freud and the Scene of Writing", p. 90) 1

Ese texto de Derrida, que cito por la versión en inglés, señala que una "fiel" traducción de los sueños es imposible a dos niveles: primero, al nivel de transcripción en una página escrita del sueño expresado oralmente por el paciente. Es imposible trasmitir en un lenguaje diferente, en este caso, lenguaje escrito, la "sonoridad", y por lo tanto, la "materialidad" de la expresión oral. Continúa Derrida: p. 90

1 Jacques Derrida: "Freud and the Scene of Writing," Yale French Studies, (No. 48, New Haven, 1972), 
To relinquish materiality is the driving force of translation. When that materiality is reinstated, translation becomes poetry. (91)

El otro nivel de imposibilidad tiene que ver con la manera en que los pensamientos inconscientes se vuelven conscientes; o según dice Derrida:

Within the psychical apparatus there is never a relation of simple translation. (91) Es decir, hay siempre un proceso de represión. Derrida aclara que

Here again, the metaphorical concept of translation or transcription is not dangerous because it refers to writing, but because it presupposes a text which would be already there, immobile.(91)

Por eso concluye que " no hay un texto presente," no hay escritura original. Hay sólo "originary prints".

The unconscious text is already woven of pure traces, differences in which meaning and force are united; a text nowhere present, consisting of archives which are always already transcriptions.... Everything begins with reproduction.

Always already: repositories of a meaning which was never present, whose signified presence is always reconstructed by deferment, belatedly, supplementarily. (92)

Como se habrá advertido, Derrida discute en su ensayo sobre Freud un problema muy especifico de traducción. Ahora quisiera llevar un poco más adelante, o traducir, este problema a los términos de la traducción literaria. Toda traducción, aún las más aparentemente fieles, no son nunca sino aproximaciones. Son textos que son análogos pero no idénticos a los llamados "originales." Por eso pueden existir infinitas traducciones de un texto determinado, y aunque algunas puedan parecer mejores que otras, ninguna de ellas dice la última palabra. Las imposibilidades de la traducción derivan de ciertos hechos inevitables, que son paralelos a los mencionados ya por Derrida. En primer lugar, una palabra determinada tiene diferentes connotaciones y una relación diferente con otras palabras del mismo lenguaje y cultura que el más cercano equivalente, o equivalentes, en otra lengua. Por ejemplo, un problema habitual que se plantea al traducir del español al inglés es la traducción de los "cognates", o palabras afines en ambos idiomas por su origen latino. En inglés, dichas palabras tienen una connotación estrictamente culta, en tanto que en español ( in negar su origen latino) suelen ser palabras comunes. A menudo, si se traduce la palabra española por su afín inglesa, se incurre en este idioma en una expresión culta que el lector común tendrá que ir a buscar al diccionario. En cambio, si se traduce la palabra española por su equivalente anglosajón (que ya no es más de origen latino), se gana en la expresión coloquial pero se pierde la connotación culta.

En su artículo. Derrida observa que "lo que ocurre en el caso de una lengua nacional específica ocurre (...) en una gramática particular',(91).Por eso, otro nivel de dificultad,o imposibilidad, se origina en que cada persona, y por lo tanto, cada escritor o traductor está, en cierto sentido, limitado a su propia lengua y usa palabras no sólo en un contexto específico, nacional o étnico, sino en el contexto de su propia mente, de su propia y particular concepción de las cosas y de la escritura. Esto lleva a la consideración de que cada texto literario contiene su propio sistema dentro de la tradición lingỉistica y literaria en que 
se inscribe, y dentro de la obra general del escritor que lo ha producido. Borges alude precisamente a esto cuando rechaza la noción de "estética" en el prefacio de su libro de poemas, Elogio de la sombra. Refiriéndose a las estéticas dice:

En general no pasan de ser abstracciones inútiles; varían para cada escritor y aún para cada texto... ${ }^{2}$

En un trabajo muy anterior, "Las versiones homéricas," ya se había referido a estos problemas. ${ }^{3}$ Tal vez Borges sea el primer escritor moderno de América Latina que ha sugerido que lo que yo he descrito aquí como el problema del traductor es también una limitación del escritor. Es decir: así como una traducción nunca puede ambicionar ser fiel al texto que aspira a reproducir, que en este caso es otro texto escrito, ninguna escritura "original" puede ser fiel al objeto, la "realidad" que parece tratar de capturar.

Las palabras, o vocablos, que son la materia prima del escritor, funcionan dentro de un sistema que está separado del sistema de la realidad que tratan de comunicar. La actividad de las palabras es menos la de definir la realidad-las palabras sólo pueden indicar o definir otras palabras-que la de reflejar como en un espejo, la de ofrecer una imagen de "otra realidad'. A esta luz, la traducción es sólo otra reflexión más en ese interminable espejeo de imágenes que es la actividad de la escritura.

II.

Guillermo Cabrera Infante y Severo Sarduy se acercan a la novela desde puntos de vista totalmente diferentes. Sarduy se aproxima desde una "estética"' de la poesía, en tanto que Cabrera Infante parte de la tradición de lo que se ha llamado en este siglo la narrativa experimental. Pero ambos parecen compartir el punto de vista de Borges que ve la escritura como traducción. Cabrera Infante lo dice más obviamente. Baste recordar el discurso bilinguie del Maestro de Ceremonias/MC al comienzo de Tres Tristes Tigres. El primer personaje de la novela resulta ser un intérprete simultáneo, es decir: un traductor. Otro de los textos que componen la novela de Cabrera Infante es la paródica transcripción escrita de las conversaciones y sueños de una paciente ante su psiquiatra. Podría verse aquí un esbozo del problema que discutía Derrida al referirse a Freud. En la parte central de la novela se encuentran las infinitas trasmutaciones de nombres, refranes y frases hechas que practica Bustrófedon, a las que hay que sumar sus parodias de los escritores cubanos; parodias en las que él "traduce" sus respectivas escrituras para ilustrar lo que habrian dicho si hubieran escrito sobre el asesinato de Trotsky. Otras señales evidentes de la misma preocupación con la traducción son las dos-o debería decir, cuatro-versiones de la historia de $\mathrm{Mr}$. Campbell y el bastón perdido y encontrado. No sólo hay una traducción correcta y una torpe del cuento del bastón sino que se ofrecen las versiones contradictorias del Sr. y la Sra. Campbell (que en realidad noes la Sra. Campbell) en forma de notas al pie de cada una de las dos traducciones. Finalmente, está también la carta de G.C.I. (tal vez: Guillermo Cabrera Infante) sobre la traducción más torpe del cuento, y el flechazo final de Silvestre sobre un notorio error en la traducción de The Old Man and the Sea (El viejo y el mar), la novella de Hemingway, por Lino Novás Calvo. Traduttore, tradittori, en efecto.

2 Jorge Luis Borges: Elogio de la sombra(Buenos Aires: Emecé Editores, 1972), p. 10.

3 Jorge Luis Borges: Discusión. (Buenos Aires: Gleizer, editor, 1932), pp. 139-140. 
Pero la dimensión de traducción está implícita en cada página de la novela de Cabrera Infante. Al fin y al cabo, él está "traduciendo"' la lengua de la Habana de los años cincuenta no sólo por el hecho de estarla transcribiendo sobre la página escrita, sino por estarla transponiendo a través del tamiz selectivo e inevitablemente deformante de su memoria. En cierto sentido, cada lector de Tres Tristes Tigres (o de la traducción al inglés que se ha publicado con el título de Three Trapped Tigers) tiene que ser un traductor. E1 libro es básicamente bilingũe, si no multilingüe, y sus juegos de palabra desafían las fronteras nacionales.

Lo mismo ocurre con Sarduy, aunque en forma menos evidente. Sarduy también está constantemente "traduciendo", es decir: transmutando tanto el lenguaje hablado como el escrito. Los signos más visibles de esta actividad son los fragmentos de habla cubana, vieja retórica española y jerga popular, jerga de los hippies, o las citas deformadas de Góngora, de la Guide Michelin, del Naked Lunch, de William Burrough. Como el bricoleur, o arréglalo-todo que Lévi-Strauss describe en La pensée sauvage, Sarduy parece usar todo el material que encuentra a mano. Es decir: como escritor artesano, toma su habla del lenguaje, de todas las formas del lenguaje, de todas las categorías del lenguaje, mezclando términos astronómicos, biológicos, pictóricos, arquitectónicos, poéticos, comerciales, religiosos, para nombrar algunas categorias. De las ruinas de esas estructuras él levanta nuevas estructuras. Esta violación de las sepulturas culturales, como la destrucción deliberada y retruecanista del lenguaje y del significado que practica Cabrera Infante en sus juegos de palabra ("la forma más baja del ingenio"), no constituyen herejías gratuitas.

Ambos escritores han escrito libros que, de manera diferente, son libros sobre la escritura misma. Una de las declaraciones principales de Sarduy es que un texto escrito es siempre, inevitablemente, la transmutación de los textos escritos que han venido antes que él. Cabrera Infante, como Sarduy, dice que el lenguaje ya es siempre una traición, una traducción del objeto que pretende expresar. Se comunica no directamente sino metafóricamente con la "realidad"; es decir: funciona sólo dentro de su propio sistema, aunque parezca reflejar y hasta ofrecer una imagen análoga de la otra "realidad". Por lo tanto, trasladar literalmente, palabra por palabra, las novelas de estos dos escritores que expresan la evasiva, fragmentaria, no-originaria naturaleza de la escritura, sería tan inútil como impráctico. La única esperanza del traductor de alcanzar cierto tipo de fidelidad con respecto al llamado "original", consiste en recrear su sistema particular de escritura; es decir: la relación entre las palabras de ese texto, y entre los distintos niveles de escritura del texto, así como la relación entre el texto y su autor, y entre el texto y su lector.

III.

Hablar de mi verdadera experiencia al traducir Cabrera Infante y Severo Sarduy es realmente discutir dos empresas casi imposibles de vincular. Mi colaboración en la versión inglesa de Tres Tristes Tigres no fue la habitual en estos casos. La novela ya había sido traducida por Cabrera Infante y un joven poeta inglés, Donald Gardner. Mi tarea fue la de colaborar con el autor en la adaptación de un texto inglés ya existente a la forma norteamericana del inglés que prevalece de este lado del Atlántico. También debí colaborar con el autor en encontrar un estilo de humor en inglés que se acercara más al suyo en 
español. En cambio, mi tarea de traducir De donde son los cantantes y Cobra fue más convencional desde este punto de vista ya que traduje a partir de un texto original preexistente, con excepción de una de las secciones de Cobra de la que Enrico Mario Santí preparó un borrador en español para ganar tiempo en lo que se había convertido en un proceso extremadamente largo. En estas obras, mi papel fue más convencional porque aunque siendo un traductor bricoleur y usando muchos diccionarios y hasta informantes vivos (incluso Roberto González Echevarría y, naturalmente, Emir Rodríguez Monegal), yo estaba en este caso al servicio del texto y no del autor, como pasó en el caso de Cabrera Infante. Por eso, me parece adecuado examinar cada traducción por separado.

El principal problema que quedaba por resolver después de terminada la primera traducción de Tres Tristes Tigres era el de traducir el habla cubana del libro en una forma más coloquial del inglés, y por lo tanto (ya que el libro iba a ser publicado en los Estados Unidos y no en Inglaterra) una forma norteamericana. Por otra parte, el lenguaje cubano está obviamente más cerca del norteamericano que del inglés. Cuando conocí a Guillermo en Londres, él estaba luchando con las primeras secciones del libro, particularmente con pasajes como la conversación telefónica de Beba Longorio, el monólogo de Magalena Crus, la carta de Delia Doce y la jerga de Estrella en los capitulos de "Ella cantaba boleros".

Empecé a ayudar a Guillermo en estas secciones y descubrimos que al hablar, es decir: al leer en voz alta las secciones orales y al echar a sonar frases y palabras hasta que encontrásemos aquellas que sonaban mejor, pudimos resolver casi por completo ese problema. Naturalmente, ciertas cosas se "perdieron" fatalmente: la jerga cubana, o mejor habanera, es única y no corresponde a ninguna norteamericana. Pero Guillermo creyó que por mi formación lingtústica en Nueva York estaba en muchos sentidos muy cerca de la textura de la suya en La Habana. También le pareció que esa mezcla de chiste a la Carole Lombard-Groucho Marx con la general y babilónica charla neoyorkina que es mi fuerte, podía constituir una buena aproximación al original coloquio habanero. También, juntos pudimos imitar las cualidades del lenguaje negro norteamericano que corresponde al de los varios personajes negros y mulatos que aparecen en la novela.

Es claro que el valor socio-lingüistico de la novela queda inevitablemente distorsionado en la versión inglesa. La abundancia de palabras inglesas y expresiones norteamericanas que aparecen en el texto español sugieren la enorme influencia de la cultura popular norteamericana (por vía del turismo y del cine) sobre la cubana, en tanto que el español que aparece en la versión inglesa, para mantener el carácter bilingïe del libro, probablemente impresiona a la mayoría de los lectores de la traducción como mero color local. Esto nos lleva a considerar el problema principal. La traducción de este libro tenía que ser una versión nueva. Es claro que esto no se debía sólo a la naturaleza del libro sino a que el autor mismo era el principal traductor. En cierto sentido, uno de los principales mensajes de Tres Tristes Tigres es que el lenguaje oculta, antes que revela; la realidad no-verbal. Esta idea está concretamente representada por las muchas conversaciones y monólogos ide la novela en la cual hablar sirve para ocultar lo que los personajes sienten, y para ocultar la información que poseen. De manera que, si consideramos que Cabrera Infante duplica esta indomable enajenación del lenguaje por su necesariamente distorsionado intento de documentar la jerga de una Habana perdida, resulta claro que su activa participación en la 
traducción al inglés de su "traducción" original al habanero tiene como consecuencia enfatizar la existencia alienada del lenguaje. La razón es simple: el inglés, para él, como el español para mí, ya es una lengua ajena, por ser extranjera. Por lo tanto, ya que las palabras en inglés tienen un peso emocional menor, y por lo tanto pueden ser separadas de su sentido, acaban por convertirse en juguetes. El resultado es que Guillermo se sintió con mayor libertad de jugar con el inglés que con el español. Es principalmente por esta razón, pues, que Three Trapped Tigers, que contiene treinta páginas más de chistes que Tres Tristes Tigres, es una versión más que una traducción.

Mi tarea como co-traductora de esta novela-además de cambiar los bloodys en damn, los weeping en crying, y en general reemplazar un lenguaje que era demasiado formal y demasiado británico en el primero borrador - fue, pues, la de ayudar al autor a inventar en inglés lo que era esencialmente para él un juego de lenguaje. Desde el momento en que Tres Tristes Tigres está lleno de neologismos y usa una sintaxis no siempre ortodoxa, tuvimos que inventar equivalentes dentro del texto inglés a los que Guilllermo había inventado dentro del texto español. Para compensar aquellos pasajes en que había palabras inventadas o retruécanos que eran intraducibles, nos tomamos la libertad de crearlos en lugares donde no había ninguno, o sustituírlos por otros nuevos en los lugares en que no podíamos encontrar equivalentes.

Por ejemplo, para compensar ciertos juegos de palabras intraducibles, Guillermo inventó un juego que pudimos jugar con la muletilla sintáctica, "of course"'. A propósito: esta adición al texto fue hecha (para desesperación del editor) después de haber entregado el manuscrito inglés a la imprenta. Otra variante que Guillermo sugirió fue que en la portada debia indicarse que el libro habia sido traducido por Donald Gardner y yo, "in closelaboration with the author". El chiste era que al hacer de dos palabras (close y colaboration) una sola, Guillermo conseguía sugerir no sólo la "estrecha colaboración", sino el duro parto (labor, en inglés) que esa traducción había sido. Pero el editor se negó firmemente a aceptar la idea, insistiendo que el texto de la portada era de su dominio exclusivo. De manera que Guillermo tuvo que aceptar la solución de "in collaboration" en vez del juego que había inventado. Pero para volver a los "of course", transcribiré ahora una frase de la carta que él me mandó entonces y en la que sugiere esta idea:

...a partir de Mirrormaze, Todos los of courses deben ser variados en Of coarse, off course, of courts, off courts, of corpse, of corset, of Corpus, of Corsican, off chorus, off curves, of corks, of Kosher, off Kursk, of source, (and so on), taking good care that it makes nonsense when you make the substitution... ${ }^{4}$

Mi tarea fue, pues, completar esta misión desesperada y colocar los "of course" a lo largo de todo el libro. Por ejemplo, en la página 153 del original, que corresponde a "La casa de los espejos", Livia dice: "Ella viene del Norte, hijo, del Cánada (Dry, tú sabes)". Aquí, parte del chiste es que Livia está haciendo un retruécano bilingüe. Naturalmente, que al traducirse la frase al inglés, el retruécano pierde su (no puede resistir la tentación) tonicidad. Este lugar era, por lo tanto, ideal para introducir uno de los distorsionados "of courses". A hora se puede leer en la página 154 de "Mirrormaze": "She comes from the

${ }^{4}$ Carta de Guillermo Cabrera Infante a la autora, fechada en Londres, mayo, 1970. 
north, honey, from Canada (Dry, of corks)., 5

En otra ocasión, el proceso inverso ocurrió. En el capítulo llamado "Algunas revelaciones', que incluye muchas categorías de gente famosa y contiene muchos chistes multi-lingües que son intraducibles, yo sugerí otra categoría que contuviese principalmente chistes ingleses y compensase por lo tanto por algunos de los chistes españoles y cubanos que eran intraducibles. Los chistes de esta sección, que titulé "Famous Books and Authors', son infortunadamente : muy primarios (id est=deescuela primaria), e incluyen horrores como Off the Cliff por Hugo Furst. Guillermo se apoderó de la idea y la elaboró con un material felizmente más sofisticado, de manera que en la página 288 de la traducción, hay una lista llamada "Famous in Books or in Famous Books" que incluyen Crime and Puns by Bustrofedon Dostowhiskey, Under the Lorry, by Malcolm Volcano, Comfort of the Season, by Gore Vidal Sassoon, etcétera, etcétera.

Nuestro propósito básico era recrear el sistema del texto original y por lo tanto teníamos que inventar chistes que despertaran asociaciones familiares al lector inglés, de la misma manera que los chistes en el "original" tenían eco en el lector hispánico. Y también, de la misma manera que ciertas cosas parecían insolubles al lector hispánico, también era necesario retener el misterio de muchas de las alusiones en la traducción. Pero, una vez más conviene insistir, esta traducción es una versión o recreación no sólo en el sentido metafórico de que toda traducción lo es, pero también en el sentido concreto de que muchos cambios textuales fueron efectuados en el nuevo libro. Por otra parte, no se crea que frívolamente descuidamos el 'original'. Para empezar, corregimos muchos errores elementales de traducción que podían encontrarse en la primera versión (" guardar" había sido consistentemente traducido por "'mirar" en vez de "to keep", ya que había sido confundido con la palabra italiana, "guardare"). También Guillermo y yo devolvimos al texto, al traducirlo, mucho del lenguaje pornográfico que había sido censurado en la versión española. De esta manera, llegamos a estar más cerca de la intención original del autor. Alguien alegó hace poco que las palabras "fuertes" de la traducción son más fuertes y numerosas que las del original. Esto ha ocurrido, en parte, por lo que ya he dicho. Gullermo quería producir en inglés el efecto que los censores españoles no le habían dejado producir. Además, la persona que objetó la fuerza de las expresiones en inglés era, naturalmente, de habla inglesa. Para ella, las palabras gruesas en inglés son más fuertes, en contenido emocional, que las de una lengua extranjera. Pero sin duda, para un lector hispánico, que pudiera leer la versión original sin el beneficio de la censura, el mismo efecto se produciría. La verdad es que, por todos estos motivos, la versión en inglés usa palabras más fuertes y las usa más habitualmente que el original. Tal vez influya en esto el hecho de que Guillermo se siente más libre de usarlas en una lengua extranjera.

IV.

Al discutir la traducción de su novela, Cabrera Infante dijo en un artículo publicado en Review: "Writers rush in where translators fear to try." ${ }^{\prime \prime}$ Si Three Trapped Tigers es

${ }^{5}$ Guillermo Cabrera Infante: Tres Tristes Tigres (Barcelona: Seix Barral, 1967). Guillermo Cabrera Infante: Three Trapped Tigers. (New York; Harper and Row , 1971).

6 Guillermo Cabrera Infante: “Epilogue for Late (n)t Readers,"' Review 1971-72 (New York:Center for Inter-American Relations), p. 27. 
una traducción abierta, principalmente fiel al sistema de la novela original que es, sin duda, una opera aperta, mis traducciones de Sarduy son mucho más "fieles". Es decir: son todo lo fieles que es posible ya que Sarduy es casi tan intraducible como Cabrera Infante. Una vez más, la última fidelidad a la que me estoy refiriendo es al sistema del texto, a su textura. El sistema de Tres Tristes Tigres es, básicamente, la traducción: cada frase es, en cierto sentido, traducción de algo escrito antes, o dicho antes. Me gustaría considerar el sistema de Cobra-la última de las novelas de Sarduy que he traducido - también como un sisterna de traducción, en el que cada palabra es una trasmutación, una repercusión, para usar una imagen de Sarduy, de algo distinto.

Pero la imagen fundamental de la escritura como traducción en Sarduy tiene una significación ligeramente distinta que en Cabrera Infante. En tanto que Cabrera Infante dice que es imposible ser fiel a la realidad "original", verbal o no-verbal, Sarduy sugiere más claramente que es imposible ser fiel al original porque no hay original. Volviendo al ensayo de Derrida sobre Freud, encontramos que allí se dice: "Everything begins with reproduction." -En este sentido, Sarduy (como ya he dicho) puede ser considerado una suerte de bricoleur. En La pensée sauvage, Lévi-Strauss presenta el bricolage, como el̉ discurso que corresponde a su propio método de investigación y de escritura sobre su investigación. El describe al bricoleur como una figura prototípica que jugó y juega una función en las sociedades llamadas "primitivas" y que todavía puede ser encontrado, en esta forma " "primera", en las sociedades "modernas"' de hoy. ${ }^{7}$ El bricoleur es alguien que usa lo que tiene a mano, los instrumentos que encuentra a su disposición. En Cobra se encuentra un ejemplo de este tipo de bricolage en el uso repetido de la imagen de la "pacotilla", que abunda en la vida de la India y que es con la que ellos construyen sus íconos religiosos. También se encuentran en Cobra alusiones a los instrumentos del bricoleur (lo que ya está alli, y no lo que se fabrica especialmente), al hablar de los objetos surrealistas "encontrados", o de las ropas "ready-to-wear".

Porque como dice Lévi-Strauss, esos instrumentos que usa el bricoleur no "han sido especialmente concebidos para la operación en que ahora son usados y a la que uno trata, por medio de sucesivas pruebas, de adaptarlos (...), o trata de usar varios de ellos al mismo tiempo, aún si su forma y origen son heterogéneos...",

En el capítulo de Cobra titulado "Enana blanca", se puede encontrar un ejemplo de este esfuerzo del mago para cambiar a Pup por medio de la "nieve." y de los conos de cobre. En otro pasaje, titulado "La conversión", el Dr. Katzob practica una operación quirúrgica en Cobra por medio de instrumentos tan inesperados como las figuras de la retórica.

Sarduy hace al viejo concepto de "literatura" lo que Lévi-Strauss hace al de la antropología tradicional. O, de la misma manera que Lévi-Strauss cuestiona las tópicas dicotomías (Naturaleza y Cultura, etc.), Sarduy cuestiona las oposiciones tradicionales que existen entre Autor y Plagiario. En su comentario a Lévi-Strauss, Derrida ha indicado que aquél usa la distinción entre Naturaleza/Cultura para demoler el viejo aparato que esa misma distinción había levantado. "Once the limit of the nature/culture opposition is felt, one might want to question systematically and rigorously the history of these concepts',

7 Claude Lévi-Strauss: The Savage Mind(The University of Chicago Press, Illinois, 1970), cap. I. 
Lévi-Strauss lo hace al conservar " in a field of empirical discovery all these old concepts, while at the same time exposing here and there their limits, treating them as tools which can still be of use." (JD, 254) ${ }^{8}$

Sarduy, como Lévi-Strauss, hace estallar los viejos conceptos para usar luego sus " "uinas". Así, Lévi-Strauss usa pedazos de antropología, filosofía e historia, para destruír esas disciplinas y construír una disciplina propia, en la cual continúa su tarea de destrucción y recomposición, ya que él hasta usa fragmentos de sus propios'escritos para contradecirlos y hacer otros, opuestos. Sarduy usa citas apolilladas de todas las "literaturas"' para tejer una nueva estructura, el texto, Cobra. Pero también produce Cobra sobre la base de repetidas y/o transformadas citas de la misma Cobra, rompiendo una y otra vez el viejo discurso. Pero en esa repetición, aún si una palabra es repetida, resulta diferente, porque ahora está en un lugar distinto y tiene una relación distintalcon las otras palabras que la rodean. Sarduy reduce constantemente a ruinas el viejo concepto marmóreo de la paternidad literaria y de la fidelidad al autor, borrando la diferencia entre lo que es original y lo que es copia.

Como ya he sugerido, tal vez la manera en que Sarduy revela el sistema nó-original y, en cierto sentido, de bricolage, de su texto, y de la escritura en general, es por medio de lo que aparece visiblemente como un proceso de flujo y reflujo, de funciones que se repiten y cambian a través de todo el texto. Me gustaría dar un ejemplo concreto tomado de un problema de traducción que pude resolver parcialmente siguiendo el sistema del texto. Me refiero a la repetición de la palabra, "opio", y al juego de palabras entre "apio" y "opio" que ocurre a lo largo del texto. ${ }^{9}$

La primera vez que aparece una referencia a la droga, "opio" (p. 19), ya está en la forma disimulada, mutada, de "apio" que denota la conocida legumbre pero sugiere, por cambio de una sola vocal inicial, la droga. También, la misma materialidad de la palabra "apio" con respecto a "opio" (la "a" por la "o"), sugiere que se trata de otro de los muchos juegos anagramáticos que aparecen en el texto de Cobra. Si hubiera traducido "apio" literalmente por "celery", hubiera eliminado toda posibilidad de recuperar las "repercusiones" que el juego apio/opio logra en español. Por lo tanto, lo que había que conseguir era una palabra que fuera similar a "opio" o que sugiriera el mismo sentido de "opio" y fuera, al mismo tiempo, una palabra inocente; es decir: una palabra que indicara normalmente un objeto que no tiene nada que ver con las drogas. Pero, al mismo tiempo, como el "apio" del original, que es considerado popularmente como afrodisiaco, tendria que ser una palabra ambigua, a la vez inocente y sugestiva. "Poppy" y "opium" podría haber sido una solución: la amapola es a la vez una flor común, símbolo para los ingleses del día del Armisticio 1918, y una de las fuentes del opio. Sin embargo, y del punto de vista verbal, "poppy" y "opium" son mucho más distintas que "apio" y "opio". Por otra parte, la amápola no es tan ambigua en su relación con el opio ya que es

\footnotetext{
8 Jacques Derrida: "Structure, Sign, and Play in the Discourse of the Human Sciences," The Structuralist Controversy, ed. by Macksey and Donato (The Johns Hopkins Press, Baltimore, 1970), p. 255.

${ }^{9}$ Severo Sarduy: Cobra (Buenos Aires: Sudamericana, 1972); English translation: Cobra (New York: Dutton, 1975).
} 
una de sus fuentes, como he dicho. "Hop" podría haber sido otra posibilidad: es una palabra del slang que significa opio, tiene dos letras en común con ésta, y sugiere el inocente verbo, "to hop' (saltar). Empero, ajustar un verbo en lugar de un nombre habría resultado incómodo, además de que "hop", como "poppy", tiene un sonido y grafía diferente de la de "opium". Me pareció que iba a ser imposible encontrar dos palabras que sugirieran lo mismo que "apio" y "opio", y que difiriesen sólo en una letra. Entonces, a cierta altura de mi búsqueda, se me ocurrió que un juego de palabras era posible con cocaina, que en muchos aspectos es similar al opio, y la Coca-Cola. En inglés, se dice generalmente "coke" por Coca-Cola; y también se usa en slang la expresión" coke" por cocaína. La Coca-Cola tiene la ventaja de ser un producto a la vez inocente y al mismo tiempo algo siniestro ya que, como el opio o la cocaína, es objeto de una explotación internacional. La palabra "coke" tiene la ventaja de ser bastante similar en sonido y grafía a la palabra inglesa, cocaine: tienen tres letras en común y pueden ser, como "apio", un anagrama parcial de Cobra. ${ }^{10}$ Por lo tanto, en la página 8 , de la traducción, convertí al "traficante de apio" del original en "bottling coke without a license"' ("embotellando Coca-Cola sin la licencia debida"). Ambas expresiones sugerían, en distinta medida, la contradicción que el texto original indicaba al calificarla irónicamente de "transgresión benigna". En la página 73 del texto español y en la 52 de la traducción, "apio' y "coke", reaparecen. "A pio", máscara del "opio", es utilizado aqui en relación con sus poderes curativos y su capacidad de estimular el crecimiento. El médico prescribe a la enana blanca la "nieve" que aquí equivale a un "alcaloide de apio"' porque, como él mismo explica, cuando algunos pastores lo utilizaron para curar la lepra blanca de sus ganados, descubrieron que, además de causar un sueño profundo, hacía crecer a los animales. Hay incluso un juego más complicado con las cualidades del opio, de manera que haré una pausa para citar la descripción que ofrece la Enciclopedia Británica del opio y la cocaína. ${ }^{11}$ El opio es un jugo, extraído de la amapola, que es blanco al principio, pero se vuelve negro en contacto con el aire. El principal principio activo del opio es el alcaloide morfina. Los usos del opio pueden ser medicinales; es decir, bajo forma de morfina puede ser utilizado como calmante. Pero también puede ser fumado, comido, bebido, e inyectado como droga ilícita. Sirve, como se sabe, para la manufactura de heroína. Es, por lo tanto, una sustancia extremadamente metamórfica. La amapola puede cultivarse en muchos lugares del planeta, y lo es en distintas zonas del Oriente. Usada en forma ilícita, la droga produce mareos, sueño, y finalmente, efectos comatosos.

La cocaína no tiene tantas formas y usos como el opio, pero tiene ciertas cualidades en común con éste, y sirve para casi todos los fines que la palabra "opio"' tiene en el texto. La cocaína es también blanca, pero no pierde su blancura en contacto con el aire; es un alcaloide cristalino que es extraído de la hoja de una planta que crece principalmente en Bolivia y en Perú, aunque también en Indonesia. Puede ser utilizada médicamente como anestesia local ya que interrumpe la trasmisión de dolor por medio de los nervios. A parece en forma pulverizada y por eso es llamada "nieve" y es, por lo general, absorbida por la

10 En una entrevista, Sarduy ha explicado algunos significados de la palabra "Cobra"'. Cf. Emir Rodriguez Monegal: "Conversación con Severo Sarduy,"'Revista de Occidente(No.93, Madrid), diciembre 1970, pp. 317321.

11 Encyclopaedia Britannica, vols. 5, p. 994, y 16, pp. 993-996 (Chicago: William Benton, 1971). 
nariz. El opio, por otra parte, aparece ya en forma líquida, o coagulada en grumos. Como droga de uso ilegal, los efectos de la cocaina son casi los opuestos del opio ya que causa insomnio, pérdida del apetito, náuseas, debilitamiento y convulsiones. La Enciclopedia concluye en tono moral que el adicto termina por convertirse en un ser irritable, quejicoso, patético.

En el párrafo de Cobra que estamos comentando (p. 73), las características del opio que juegan algún papel son su blancura, en relación con la enana blanca y con la lepra blanca, y el efecto de producir sueño. Por lo tanto, este permite el juego de palabras con Morfeo, morfina, morfea. El hecho de que el opio puede ser inyectado y de que crece por lo general en el lejano Oriente son también significantes aquí. Al usar la palabra "coke", eliminé la alusión a la morfina, y por lo tanto a toda la serie Morfeo/morfea, y también el efecto narcótico. Este cambio textual sacrificó también la relación, ya mencionada, con la palabra "morfológica". Pero, por otra parte, además de aludir al ya establecido juego de palabras entre "coke" y "cocaine", consegui mantener la relación con el sinónimo, "nieve", que aparece a lo largo de toda la novela, y con la droga, en este caso, la cocaína. "Nieve" es, por otra parte, la más usada palabra de slang para cocaína, aunque puede significar también heroína. Además, ya se ha visto que la cocaína también crece en el Oriente aunque no en forma tan general. Por lo tanto, la palabra "cocaína" no elimina la atmósfera oriental que es fundamental en el texto.

En la página 194 del texto español, "opio" aparece en su sentido de calmante durante una operación quirúrgica, pero esta vez la palabra no es aludida a través de la metáfora del "apio"' sino en su forma normal. Yo podria, por lo tanto, haberla dejado en el texto inglés pero como había decidido sustituírla por cocaína antes, me pareció obviamente necesario para mantener el sistema de alusiones, usar cocaína aquí también (ver página 78 de la versión en inglés). En este caso, la cocaína puede ser considerada como un sustituto lógico ya que en su forma medicinal puede ser utilizada para evitar el dolor.

En la página 114 del texto español que corresponde a la 87 del inglés, "opio" es ofrecido a un hombre que está sufriendo la tortura de los Cien Cortes. Esta misma escena, por otra parte, había sido utilizada antes por Cortázar en Rayuela, y más tarde minuciosamente desarrollada por Salvador Elizondo, en Farabeuf. ${ }^{12}$. Una vez más, sustituí "opio" por "cocaina", aunque idealmente y si se considera el contexto chino, "opio" habria sido una traducción más legítima.

V.

Espero haber demostrado con este ejemplo lo que quiero decir cuando hablo de traducir el sistema del texto. Al sustituír "apio" y "opio" por "coke" y "cocaine", he tratado de recapturar un sistema bastante complicado de alusiones y unas relaciones no sólo de significados sino también de sonidos. En Cobra no hay un código, permanente e inmóvil. Como la palabra "opio", Cobra, el texto, está perpetuamente cambiando de significado así como de forma. Por lo tanto, la única clase de traducción posible es la que intente trasmitir ese código elusivo. Lo mismo pasa en Tres Tristes Tigres, aunque alli el código sea otro. Toda traducción es reproducción. y re-escritura.

New. York University

SUZANNE JILL LEVINE

12 Severo Sarduy:Escrito sobre un cuerpo(Buenos Aires: Sudamericana, 1969), pp. 9-30. 
